\title{
X-ray imaging of the dynamic magnetic vortex core deformation
}

\author{
A. Vansteenkiste ${ }^{1 \star}$, K. W. Chou ${ }^{2}$, M. Weigand ${ }^{3}$, M. Curcic ${ }^{3}$, V. Sackmann ${ }^{3}$, H. Stoll ${ }^{3}$, T. Tyliszczak ${ }^{2}$, \\ G. Woltersdorf ${ }^{4}$, C. H. Back ${ }^{4}$, G. Schütz ${ }^{3}$ and B. Van Waeyenberge ${ }^{3}$
}

\begin{abstract}
Magnetic thin-film square- or disc-shaped nanostructures with adequate dimensions exhibit a magnetic vortex state: the magnetization vectors lie in the film plane and curl around the structure centre. At the very centre of the vortex, a small, stable core exists where the magnetization points either up or down ${ }^{1,2}$. The discovery of an easy core reversal mechanism ${ }^{3}$ did not only open the possibility of using such systems as magnetic memories, but also initiated the fundamental investigation of the core switching mechanism itself ${ }^{4-15}$. Theoretical modelling predicted that the reversal is mediated by the creation and annihilation of a vortex-antivortex pair ${ }^{3,4,16}$, but experimental support has been lacking until now. We used high-resolution time-resolved magnetic X-ray microscopy to experimentally reveal the first step of the reversal process: the dynamic deformation of the vortex core. In addition, we have measured a critical vortex velocity above which reversal must occur 5,17 . Both observations support the previously proposed reversal mechanism.
\end{abstract}

Depending on the material type and thickness, the vortex core diameter is typically only 10 (ref. 18) to $25 \mathrm{~nm}$ (ref. 19). Although the core is very small, it significantly affects the dynamics as it gives rise to the so-called vortex gyration mode ${ }^{20-22}$. This mode corresponds to a circular motion of the vortex around the structure centre. It was recently shown that a low-field excitation of this mode can switch the out-of-plane polarization of the core ${ }^{3}$. This was experimentally observed by determining the vortex core polarization before and after the application of short bursts of an alternating magnetic field ${ }^{3}$. The dynamic process behind the switching could not be inferred from this experiment. However, micromagnetic modelling showed that near a moving vortex core, a region appears where the magnetization acquires an out-of-plane component opposing the vortex core polarization. If this so-called vortex core deformation becomes so strong that it points fully out of the sample plane, a vortex-antivortex pair is nucleated. At this point, the switching is initiated, as the antivortex rapidly annihilates with the original vortex, leaving behind only the newly created vortex core with an opposite polarization ${ }^{3,4,16}$. This annihilation process involves a magnetic singularity, which is necessary for the polarization reversal ${ }^{23}$.

Apart from micromagnetic simulations, the dynamic core deformation had already been included in theoretical calculations by Novosad et $a l^{24}$. Its origin and relevance for the switching process were investigated by Yamada et al. ${ }^{17}$ and by Guslienko et al. ${ }^{5}$. These authors showed that near a moving vortex, a strong out-of-plane 'kinetic' term in the effective field appears. It is this field that pushes the magnetization towards the opposite direction of the core polarization, causing the dynamic deformation.

As the effective out-of-plane field is proportional to the velocity of the vortex movement ${ }^{17}$, a critical velocity must exist at which a vortex-antivortex pair is created and core switching will occur ${ }^{17}$. The critical velocity is predicted to be independent of the strength and type of the applied excitation and to depend only on the exchange parameter $A$ (ref. 5). Calculated critical velocities for typical permalloy $\left(\mathrm{Ni}_{80} \mathrm{Fe}_{20}\right)$ systems range from 250 (ref. 17) to $320 \mathrm{~m} \mathrm{~s}^{-1}$ (ref. 5).

We have used time-resolved magnetic scanning transmission $\mathrm{X}$-ray microscopy (STXM) to image the detailed dynamics of the out-of-plane component of the magnetization in permalloy nanostructures. A spatial resolution of about $30 \mathrm{~nm}$ and a temporal resolution of $100 \mathrm{ps}$ could be reached (see the Methods section). Micromagnetic simulations (see the Methods section) yield a vortex core diameter of $25.4 \mathrm{~nm}$ (full-width at half-maximum, averaged over the layer thickness) for the investigated structure. Although this is smaller than the lateral resolution of the microscope, it can still be resolved but appears smeared out ${ }^{19}$.

Figure 1a,b shows STXM images of the centre portion of a $500 \mathrm{~nm} \times 500 \mathrm{~nm} \times 50 \mathrm{~nm}$ permalloy square structure. The structure is excited with a continuous in-plane radiofrequency magnetic field oscillating at a frequency of $562.5 \mathrm{MHz}$, close to the eigenfrequency of the gyrotropic mode ${ }^{25}$. Under these conditions, a steady-state gyration of the vortex is obtained. To remove the non-magnetic contrast contributions, reference images recorded at a $180^{\circ}$ phase shift of the radiofrequency excitation are subtracted from the respective images. In these so-called differential images, the vortex core appears twice: once from the original image, and once with inverted contrast from the reference image. The positions of these two vortex core images are mirrored with respect to the structure centre and appear well separated when the gyration radius is larger than the lateral resolution of the microscope-about $30 \mathrm{~nm}$. The stroboscopic images were recorded at eight phases of the gyration cycle, with time intervals of 222 ps between them. The last four images were then subtracted from the respective first four, and the resulting four differential images are shown. In Fig. 1a, the vortex core points up and appears as a red spot, marked with a circle. Note that the vortex core gyrates anticlockwise, as dictated by its polarization ${ }^{20}$. The blue spot in the image originates from the subtraction of the reference image, and corresponds to a negative image of the core appearing with a $180^{\circ}$ phase shift in the gyration cycle. The amplitude of the excitation here is $0.32 \mathrm{mT}$ and causes the core to gyrate with an average velocity of $(160 \pm 20) \mathrm{m} \mathrm{s}^{-1}$. This velocity was determined from the average radius $r$ of the gyration,

${ }^{1}$ Department of Subatomic and Radiation Physics, Ghent University, Proeftuinstraat 86, 9000 Gent, Belgium, ${ }^{2}$ Advanced Light Source, LBNL, One Cyclotron Road, 94720 Berkeley, California, USA, ${ }^{3}$ Max-Planck-Institut für Metallforschung, Heisenbergstr. 3, 70596 Stuttgart, Germany, ${ }^{4}$ Institut für Experimentelle und Angewandte Physik, Universität Regensburg, 93040 Regensburg, Germany. *e-mail: arne.vansteenkiste@ugent.be. 
a

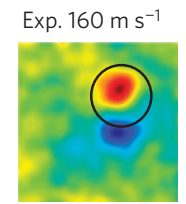

b

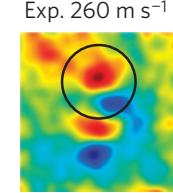

C

(no roughness), $280 \mathrm{~m} \mathrm{~s}^{-1}$
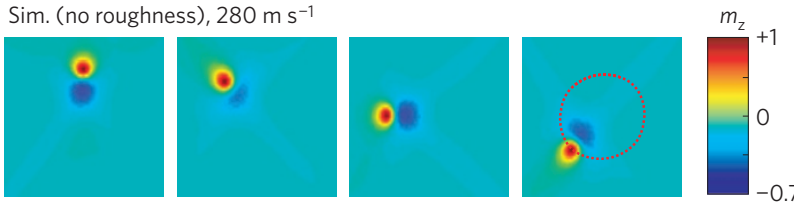

d
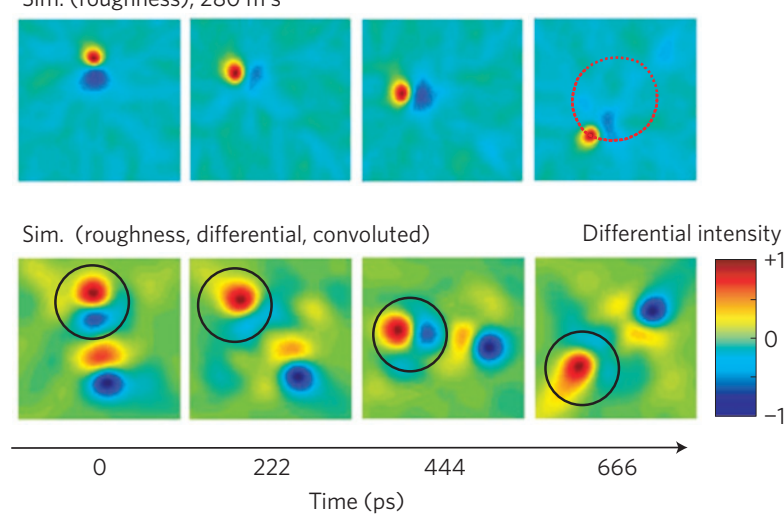

Figure 1 | Experimental and simulated images of the time-dependent out-of-plane component of the magnetization of a vortex structure.

a, Differential STXM images under a continuous $0.32 \mathrm{mT}$ radiofrequency excitation. Red/blue corresponds to the magnetization pointing up/down, respectively. The vortex core can be seen as a red spot, marked by a circle (the second spot originates from the subtraction of the reference image). b, Images of the same sample, but with a $0.89 \mathrm{mT}$ excitation. The dynamic vortex core deformation is now visible as an extra spot near the vortex core. c,d, Simulation of the steady-state vortex gyration in a permalloy structure under similar conditions as the experiments, with and without sample roughness (see the Methods section). The core trajectory is shown with a dotted line. e, Differential images generated from the simulations in d, convoluted with the experimental lateral resolution. These images can be directly compared to the experimental images in $\mathbf{b}$.

because the angular velocity $2 \pi f$ is fixed and $v=2 \pi f r$. The trajectory was thus assumed to be approximately circular, which is verified by the simulations in Fig. 1c-d.

When the excitation amplitude is increased, a larger gyration amplitude, and consequently a larger velocity, can be observed. At $0.89 \mathrm{mT}$ (Fig. 1b), the average gyration velocity is $(260 \pm 20) \mathrm{m} \mathrm{s}^{-1}$. This excitation is already very close to the switching threshold. When the field was increased to $1 \mathrm{mT}$, the vortex core polarization was found to be switching back and forth. Further investigation of the images recorded just below the switching threshold reveals an extra spot near the vortex core (also shown in Fig. 2). Having a magnetization opposite to the core, this spot is identified as the dynamic vortex core deformation. This deformation was predicted as the nucleation site of the vortex-antivortex pair when the gyration velocity reaches the threshold for core switching ${ }^{5,17}$.

Besides the direct observation of the dynamic vortex core deformation, these experiments also enable determination of the critical velocity for core switching. This was done by increasing the

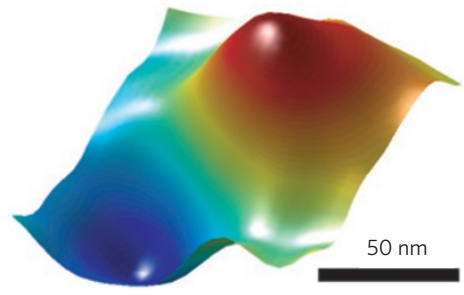

Differential intensity

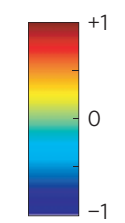

Figure 2 | Three-dimensional representation of the experimentally observed vortex core profile, generated from the marked area in the last frame of Fig. 1b. The differential intensity is proportional to the out-of-plane magnetization. Although the features are smaller than the resolution of the microscope and are therefore significantly smeared out, the bipolar nature of the vortex core profile can still be clearly observed.

excitation in small steps of maximum $12 \%$ until vortex core reversal was observed. The critical switching velocity was estimated by determining the maximum core velocity, right below the switching threshold. This procedure was repeated when the excitation frequency was detuned, away from the gyrotropic resonance where higher excitation amplitudes are necessary to achieve switching. The highest measured velocities of various samples at different excitation amplitudes are shown in Fig. 3. Even when the magnetic field amplitude required for switching varied by more than a factor of two, the core velocity just below the threshold was found to be constant within the experimental error. The vortex core velocity thus seems indeed to be the critical switching parameter, regardless of excitation amplitude and frequency.

The experimental results could be well reproduced by micromagnetic simulations. A permalloy platelet with the same dimensions as the experimentally investigated ones was simulated. In Fig. 1c, the simulated out-of-plane component of the magnetization is shown during a steady-state gyration just below the switching threshold. The vortex core deformation can be distinguished as a spot with opposite polarization next to the vortex core. The strength of the deformation is not entirely constant, but its fluctuations follow the four-fold symmetry of the square sample. To reproduce the experimental results as well as possible, simulations including a small surface roughness (see the Methods section) were carried out as well (Fig. 1d). In our evaporated thin films, such surface roughness is inevitable. The roughness did not change the underlying switching mechanism itself, but was found to only induce some extra fluctuations in the deformation profile as well as some extra out-of-plane magnetization components, as is also seen in the experimental images.

To illustrate the correspondence between the simulations and the experiments, a series of differential images was generated from the simulations with roughness (Fig. 1d). These are obtained using the same procedure as the experimental differential images (from each image, the image corresponding to a $180^{\circ}$ phase shift of the radiofrequency excitation has been subtracted), and have been convoluted with a Gaussian function corresponding to the lateral resolution of the microscope. The position and size of the deformation in these images can be seen to convincingly mimic the experimental data in Fig. 1b.

From the simulations, an instantaneous critical velocity of $300 \mathrm{~m} \mathrm{~s}^{-1}$ was found, which is well in the range of the predicted velocities $^{5,17}$. The vortex core velocity was however found to fluctuate along the trajectory. This is not due only to the periodic acceleration by the radiofrequency field, but also due to the surface roughness. Therefore, the average velocity over one period was slightly lower than the peak velocity: about $280 \mathrm{~m} \mathrm{~s}^{-1}$. This is in good agreement with the experimental average velocities, considering that the experimental values are lower limits of the actual switching threshold. The observation of both the dynamic 


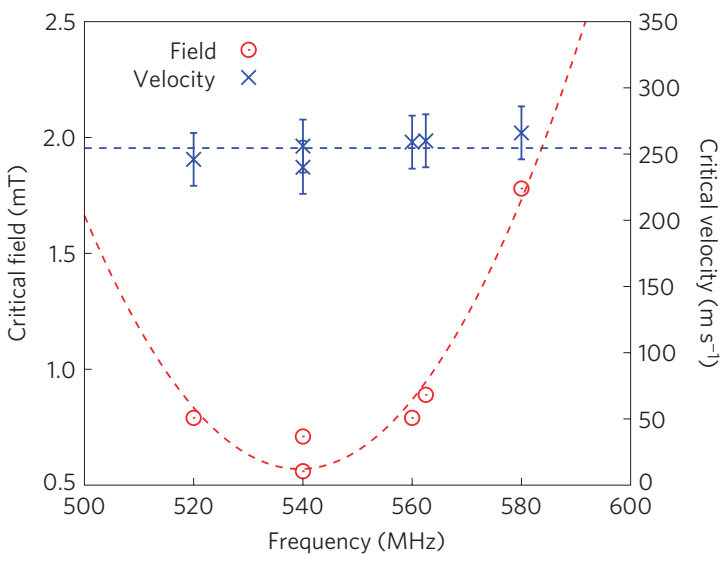

Figure 3 | Magnetic field amplitudes $B_{0}$,thr and average vortex velocities $v_{\text {thr }}$ just below the threshold for core switching, determined for different excitation frequencies $f$ near the gyrotropic resonance of three $\mathbf{5 0 0} \mathbf{~ n m} \times \mathbf{5 0 0} \mathbf{~ n m} \times \mathbf{5 0} \mathbf{~ n m}$ permalloy samples. The critical field required for switching (red points) varies by more than a factor of two when the excitation frequency is detuned away from the resonance. The critical velocity (blue points), however, remains constant within the experimental error (the standard deviation of the velocities, determined at eight different phases of the motion, rounded up). The dashed curves are a guide to the eye.

vortex core deformation and the critical vortex velocity are thus in agreement with the model for vortex core reversal through the creation and annihilation of a vortex-antivortex pair.

\section{Methods}

Experiments. High-resolution images of the vortex core dynamics in permalloy nanostructures were recorded using the scanning transmission X-ray microscope at the Advanced Light Source (beamline 11.0.2; ref. 26). This microscope has a resolution of about $30 \mathrm{~nm}$. The presented images were slightly smoothed to reduce the noise, without losing significant information (the pixel size was $5 \mathrm{~nm}$, well below the resolution of the microscope). No smoothing was applied during the quantitative analysis of the data. The investigated nanostructures were square-shaped and patterned from an evaporated permalloy film on top of a $2.5-\mu \mathrm{m}$-wide and $150-\mathrm{nm}$ thick $\mathrm{Cu}$ stripline used for the magnetic excitation. The magnetization is imaged using the X-ray magnetic circular dichroism effect ${ }^{27}$. By orienting the sample plan perpendicular to the photon beam, the out-of-plane component of the magnetization is measured, enabling direct imaging of the vortex core ${ }^{19}$. A radiofrequency current is transmitted through the stripline underneath the magnetic structures and induces an in-plane radiofrequency magnetic field $B(t)=B_{0} \sin (2 \pi f t)$. By synchronizing the excitation with the X-ray flashes of the synchrotron, stroboscopic images of the moving vortex core can be recorded. The time resolution of these images is limited by the width of the photon flashes to about $100 \mathrm{ps}$.

Simulations. A $500 \mathrm{~nm} \times 500 \mathrm{~nm} \times 50 \mathrm{~nm}$ platelet was simulated with a cell size of $3.9 \mathrm{~nm} \times 3.9 \mathrm{~nm} \times \pm 12.5 \mathrm{~nm}$. Typical material parameters for permalloy were used: saturation magnetization $M_{\mathrm{s}}=800 \times 10^{3} \mathrm{~A} \mathrm{~m}^{-1}$, exchange constant $A=13 \times 10^{-12} \mathrm{~J} \mathrm{~m}^{-1}$, damping parameter $\alpha=0.01$, anisotropy constant $K_{1}=0$. An excitation of $1.9 \mathrm{mT}$ at $562.5 \mathrm{MHz}$ was applied, which is just below the switching threshold. The AMUMAG micromagnetic package, an in-house-developed three-dimensional finite-element code, was used to solve the Landau-Lifshitz equation ${ }^{28}$. The source code is available at http://code.google.com/p/amumag. The fast multipole method ${ }^{29}$ was used to calculate the magnetostatic fields, assuming constant magnetization in each of the hexahedral cells. The simulations did not include thermal fluctuations. To match the realistic sample geometry as closely as possible, a small random surface roughness was introduced (r.m.s. amplitude $\approx 5 \mathrm{~nm}$, correlation length $\approx 20 \mathrm{~nm}$ ).

Received 24 October 2008; accepted 20 February 2009; published online 29 March 2009

\section{References}

1. Feldtkeller, E. \& Thomas, H. Struktur und Energie von Blochlinien in dünnen ferromagnetischen Schichten. Phys. Kondens. Mater. 4, 8-14 (1965).

2. Raabe, J. et al. Magnetization pattern of ferromagnetic nanodisks. J. Appl. Phys. 88, 4437-4439 (2000).

3. Van Waeyenberge, B. et al. Magnetic vortex core reversal by excitation with short bursts of an alternating field. Nature 444, 461-464 (2006).
4. Hertel, R., Gliga, S., Fähnle, M. \& Schneider, C. M. Ultrafast nanomagnetic toggle switching of vortex cores. Phys. Rev. Lett. 98, 117201 (2007).

5. Guslienko, K. Y., Lee, K. S. \& Kim, S. K. Dynamic origin of vortex core switching in soft magnetic nanodots. Phys. Rev. Lett. 100, 027203 (2008).

6. Kim, S. K., Choi, Y. S., Lee, K. S., Guslienko, K. Y. \& Jeong, D. E. Electric-current-driven vortex-core reversal in soft magnetic nanodots. Appl. Phys. Lett. 91, 082506 (2007).

7. Kim, S. K., Lee, J. Y., Choi, Y. S., Guslienko, K. Y. \& Lee, K. S. Underlying mechanism of domain-wall motions in soft magnetic thin-film nanostripes beyond the velocity-breakdown regime. Appl. Phys. Lett. 93, 052503 (2008).

8. Gliga, S., Yan, M., Hertel, R. \& Schneider, C. M. Ultrafast dynamics of a magnetic antivortex: Micromagnetic simulations. Phys. Rev. B 77, 060404 (2008).

9. Gliga, S., Hertel, R. \& Schneider, C. M. Flipping magnetic vortex cores on the picosecond time scale. Physica B 403, 334-337 (2008).

10. Gliga, S., Hertel, R. \& Schneider, C. M. Switching a magnetic antivortex core with ultrashort field pulses. J. Appl. Phys. 103, 07B115 (2008).

11. Liu, Y. W., He, H. \& Zhang, Z. Z. Spin-torque-driven vortex dynamics in a spin-valve pillar with a perpendicular polarizer. Appl. Phys. Lett. 91, 242501 (2007).

12. Liu, Y., Gliga, S., Hertel, R. \& Schneider, C. M. Current-induced magnetic vortex core switching in a Permalloy nanodisk. Appl. Phys. Lett. 91, 112501 (2007).

13. Lee, K. S., Guslienko, K. Y., Lee, J. Y. \& Kim, S. K. Ultrafast vortex-core reversal dynamics in ferromagnetic nanodots. Phys. Rev. B 76, 174410 (2007)

14. Xiao, Q. F. et al. Dynamics of magnetic vortex core switching in Fe nanodisks by applying in-plane magnetic field pulse. J. Appl. Phys. 102, 103904 (2007).

15. Kravchuk, V. P., Sheka, D. D., Gaididei, Y. \& Mertens, F. G. Controlled vortex core switching in a magnetic nanodisk by a rotating field. J. Appl. Phys. 102, 043908 (2007).

16. Gaididei, Y. B., Kravchuk, V. P., Sheka, D. D. \& Mertens, F. G. Switching phenomena in magnetic vortex dynamics. Low Temp. Phys. 34, 528-534 (2008),

17. Yamada, K. et al. Electrical switching of the vortex core in a magnetic disk. Nature Mater. 6, 269-273 (2007).

18. Wachowiak, A. et al. Direct observation of internal spin structure of magnetic vortex cores. Science 298, 577-580 (2002).

19. Chou, K. W. et al. Direct observation of the vortex core magnetization and its dynamics. Appl. Phys. Lett. 90, 202505 (2007).

20. Huber, D. L. Equation of motion of a spin vortex in a two-dimensional planar magnet. J. Appl. Phys. 53, 1899-1900 (1982).

21. Argyle, B. E., Terrenzio, E. \& Slonczewski, J. C. 1984 Digests Of Intermag '84. International Magnetics Conference (cat. No. 84ch1918-2) 350-350 (1984).

22. Guslienko, K. Y. et al. Eigenfrequencies of vortex state excitations in magnetic submicron-size disks. J. Appl. Phys. 91, 8037-8039 (2002).

23. Thiaville, A., Garcia, J. M., Dittrich, R., Miltat, J. \& Schrefl, T. Micromagnetic study of Bloch-point-mediated vortex core reversal. Phys. Rev. B 67, 94410 (2003).

24. Novosad, V. et al. Magnetic vortex resonance in patterned ferromagnetic dots. Phys. Rev. B 72, 024455 (2005).

25. Vansteenkiste, A. et al. Influence of domain wall pinning on the dynamic behaviour of magnetic vortex structures: Time-resolved scanning $\mathrm{x}$-ray transmission microscopy in NiFe thin film structures. Phys. Rev. B 77, 144420 (2008).

26. Kilcoyne, A. et al. Interferometer-controlled scanning transmission $\mathrm{x}$-ray microscopes at the advanced light source. J. Synchrotron Radiat. 10, 125-136 (2003).

27. Schütz, G. et al. Absorption of circularly polarized x-rays in iron. Phys. Rev. Lett. 58, 737-740 (1987).

28. Landau, L. \& Lifshitz, E. Theory of the dispersion of magnetic permeability in ferromagnetic bodies. Phys. Z. Sowietunion 8, 153-169 (1935).

29. Visscher, P. B. \& Apalkov, D. M. Charge-based recursive fast-multipole micromagnetics. Physica B 343, 184-188 (2004).

\section{Acknowledgements}

Financial support by The Institute for the promotion of Innovation by Science and Technology in Flanders (IWT-Flanders) and by the Research Foundation Flanders (FWO-Flanders) through the research grant 60170.06 is gratefully acknowledged. The Advanced Light Source is supported by the Director, Office of Science, Office of Basic Energy Sciences, of the US Department of Energy.

\section{Author contributions}

Analyses and micromagnetic simulations: A.V.; experiments: A.V., K.W.C., M.W., M.C., V.S., H.S., T.T., B.V.W.; writing the paper: A.V., K.W.C., G.W., C.H.B., H.S., B.V.W.; sample preparation: G.W., C.H.B.; project planning: H.S., B.V.W., G.S

\section{Additional information}

Reprints and permissions information is available online at http://npg.nature.com/ reprintsandpermissions. Correspondence and requests for materials should be addressed to A.V. 